Review

\title{
Parenteral Nutrition: Review of Recent American Society for Parenteral and Enteral Nutrition Publications
}

Phil Ayers ${ }^{*}$, Megan Harlow, LeAnn Scott

Mississippi Baptist Medical Center, Jackson, MS; E-Mails: George.Ayers@BMHCC.org;

Megan.Harlow@BMHCC.org; LeAnn.Scott@BMHCC.org

* Correspondence: Phil Ayers; E-Mail: George.Ayers@BMHCC.org

Academic Editor: Jennifer Keogh

Special Issue: Feature Papers of Recent Progress in Nutrition 2021

Recent Progress in Nutrition

2022, volume 2 , issue 1

doi:10.21926/rpn.2201002
Received: December 30, 2021

Accepted: February 09, 2022

Published: February 15, 2022

\begin{abstract}
\section{Keywords}

Parenteral nutrition; lipid injectable emulsion; filters; photoprotection
\end{abstract}

Parenteral nutrition (PN) is a complex medication administered to adult, pediatric, and neonatal patients. PN is recognized as a high-alert medication by the Institute for Safe Medication Practices (ISMP). The American Society for Parenteral and Enteral Nutrition (ASPEN) has published several key recommendations regarding safe use of PN. This manuscript will review several of the important PN papers published in 2020-2021 by ASPEN.

\section{Introduction}

Parenteral nutrition (PN) is a complex medication administered to adult, pediatric, and neonatal patients. PN is recognized as a high-alert medication by the Institute for Safe Medication Practices (ISMP) [1]. Complications may occur because of the complexity of PN itself or due to failure to

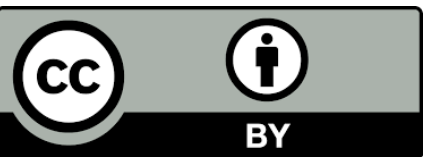

(C) 2022 by the author. This is an open access article distributed under the conditions of the Creative Commons by Attribution License, which permits unrestricted use, distribution, and reproduction in any medium or format, provided the original work is correctly cited. 
adhere to important steps throughout the PN process. The American Society for Parenteral and Enteral Nutrition (ASPEN) has published several key recommendations regarding safe use of PN. This manuscript will review several of the important PN papers published in 2020-2021 by ASPEN. More in-depth information may be found in the original articles.

\section{Methods}

A review of ASPEN papers related to parenteral nutrition published in 2021 and 2021 was conducted. Search terms included parenteral nutrition, lipid injectable emulsion, filters, and administration. 5 papers were identified for inclusion in this review article.

\section{ASPEN Consensus Recommendations for Refeeding Syndrome [2]}

Due to many differences published in refeeding syndrome (RS) literature, the ASPEN Parenteral Nutrition Safety Committee and the Clinical Practice Committee assembled a task force to unify the clinical definition of RS as well as identify and propose recommendations for patients at risk for RS and for avoiding and managing RS in the adult and pediatric hospitalized patients. There was also an erratum to this paper that was published to correct an error in one of the tables published in the original article [3]. Neonates were not included in the proposed criteria due to their complexity. Additionally, the task force encourages recommendations to be used in conjunction with standards of care. The proposed criteria should be utilized in future research opportunities. Typically, the most illustrated marker of RS is hypophosphatemia. Perhaps, equally important, ions such as magnesium and potassium may also be depleted in RS. Insulin- like growth factor 1(IGF-1) and leptin are biomarkers that can also play a vital role in clinical decision making, but insufficient literature exists to recommend its routine use as predictive markers. Patients at risk for RS may include those with anorexia nervosa, mental health disorders, alcohol and substance-use disorders, bariatric surgery, bowel resections, malabsorption, starvation, and more rare military recruits as well as athletes. Other populations potentially at risk for RS include the critically ill, patients with malignancies, emergency department admissions, and renal failure. Hemodialysis dependent patients are more unlikely at risk for RS secondary to poor clearance of ions.

Diagnostic criteria for identifying patients at risk for RS are outlined as either mild, moderate, or significant risk categories. Adult patients are excluded from mild risk categorization due to low clinical significance as well as no difference in therapeutic management. Information is provided regarding initiation and advancement of PN in patients at risk for RS. Recommendations for avoidance and treatment of RS in at-risk adult and pediatric patients are outlined by initiation of calories, fluid/sodium/protein restriction, electrolytes, thiamin, multivitamins, and monitoring and long-term care. ASPEN consensus recommendations for screening, avoiding, and treating RS provide unified criteria, and further research should be conducted to determine its impact.

\section{ASPEN Lipid Injectable Emulsion Safety Recommendations, Part 1: Background and Adult Considerations [4]}

Lipid injectable emulsions (ILEs) are used in PN to provide fatty acids (FA) and energy. There are several issues with ILE use including emulsion stability, dose, and infusion tolerance. Multiple ILE products have been developed to improve safety and efficacy. Currently there are five ILE products 
available in the United States. See the referenced article for further tables and details on rational for design, key differences in composition, and dosing.

SO-ILE was originally developed to prevent essential FA deficiency (EFAD). In general, EFAD is rare with the exception of those chronically receiving PN without ILE. Some consequences of EFAD include alopecia and growth retardation. ILE use can also result in a decrease in the dextrose required for appropriate energy intake which can improve glycemic control and minimize liver function test (LFT) elevations.

As $\omega-6$ FA has been associated with inflammation and immunosuppression in critically ill patients, guidelines recommend withholding SO-ILE from the PN through the first week of therapy or limiting the weekly dose to a maximum limit of 100 grams if EFAD is suspected.

ILE use can lead to several adverse events including fat overload syndrome, elevated liver function tests (LFTs), increased risk of infections, and hypertriglyceridemia. Although allergic reactions to ILEs are rare, there are patients who have hypersensitivity to the components of the PN admixture. The most common local reaction is pruritus, however it should be noted that reactions can vary. If the patient is allergic to a specific oil in the ILE, they can be changed to another product that utilizes an alternative oil if long term PN use is required. Serum triglyceride and LFT levels should be drawn at baseline and weekly thereafter.

Factors affecting ILE safety include the risk of increased droplet size and the impediment of visual inspection due the opalescence of the product. A 1.2- $\mu \mathrm{m}$ filter should be used to prevent infusion of large fat droplets and particulates. Infusion of particles over $5 \mu \mathrm{m}$ could result in a fat embolism. Concerns about bacterial contamination have led to the recommendation for a 12-hour infusion time for ILE, however, this is extended to $24 \mathrm{~h}$ for TNAs (total nutrient admixtures) as they do not favour microbial growth.

The paper notes that ILE dose should be entered in grams per day and not be determined by the volume of the available product container. If other lipid containing preparations like propofol or clevidipine are being simultaneously administered, the dose of the ILE should be reduced accordingly.

In summary, ILE is an essential component of PN therapy. Originally SO-ILE was this source of energy, however, now there are five ILE products available in the United States. Some of these alternative products are associated with fewer side effects.

\section{Update on the Use of Filters for Parenteral Nutrition: An ASPEN Position Paper [5]}

In the United States, intravenous in-line filters are required for the administration of PN. PN filter guidelines have been issued by the Food and Drug Administration, ASPEN, the Infusion Nurses Society and PN component manufactures. A recent survey of ILE practices noted $10-20 \%$ of respondents did not adhere to current ILE filtering guidelines [6].

This paper provides a chronology of filter use recommendations from 1994-2016. It is noted that there may be confusion among clinicians regarding the use of 1.2 micron filters ILE administration.

Administration of PN, as a total nutrient admixture (amino acid, dextrose, lipids in one bag) requires a 1.2 micron filter. An illustration is provided in this paper to guide setup for the use of a 1.2 micron filter for administration of a 2 in 1 (amino acid-dextrose) admixture with ILE given as a separate infusion. The illustration shows the use of the 1.2 micron filter below the administration tubing Y-site. This should clarify the use of filters and simplify the process. 


\section{Recommendations for Photoprotection of Parenteral Nutrtion for Premature Infants: An ASPEN Position Paper [7]}

The use of PN in premature infants is an important therapeutic modality due to immature gastrointestinal tracts and risk of developing nutritional deficits in the first weeks of life. A growing body of evidence indicates that components of PN, ILE, multivitamins, amino acids and trace elements, are susceptible to oxidation when exposed to light. PN exposed to sunlight, ambient light and phototherapy can result in the production of hydrogen peroxide and organic peroxides such as lipoperoxides and ascorbylperoxides. Infants, especially preterm, are more susceptible to oxidative stress than children and adults. Bronchopulmonary dysplasia, retinopathy of prematurity, necrotizing enterocolitis and intestinal-failure-associated liver disease have been associated with oxidative stress.

This manuscript reviews studies and provides guidance on how to enhance the ability to photoprotect PN utilized in premature infants. Recommendations regarding compounding, delivery, administration and supplies are provided in the document.

\section{ASPEN Lipid Injectable Emulsion Safety Recommendations Part 2: Neonate and Pediatric Considerations [8]}

This is the second paper of a 2 part series addressing ILE and safety recommendations. The focus of the manuscript is ILE use in neonatal and pediatric patients. The nutritional needs of neonatal and pediatric patients differ from adults. These populations have greater protein and caloric needs in order to support growth and development.

An overview of ILE fatty acid composition is provided along with the importance of ILE in the prevention of essential fatty acid deficiency. Intestinal failure associated liver disease (IFALD) is a serious complication seen in premature infants and those with short bowel syndrome. IFALD is multifactorial and can be partly due to the phytosterol content of ILE. Factors such as risk of IFALD, bronchopulmonary dysplasia and retinopathy of prematurity should be considered when prescribing ILEs.

The manuscript provides a very useful summary of recommendations addressing indication, prescribing (amount/kilogram/day), order review, preparation, administration, transfer of care and monitoring of ILE in pediatric and neonatal patients.

\section{Conclusion}

$\mathrm{PN}$ is a complex medication that can cause significant harm if not prescribed appropriately. Selection of appropriate candidates and careful initiation are imperative for safe PN use.

Several new ILE products are available for adult and pediatric patients in the United States. Clinicians should select the ILE based on acuity of the patient and anticipated length of therapy.

Important recommendations regarding filtering and photoprotection should be incorporated by organizations involved in the provision of PN.

\section{Author Contributions}

All authors contributed equally to this work. 


\section{Conflict of Interest}

Dr. Ayers is a consultant and speaker for Fresenius Kabi and Vice President of the American Society for Parenteral and Enteral Nutrition (ASPEN). Dr Harlow and Dr. Scott have no conflicts of interest to disclose.

\section{References}

1. Institute for Safe Medication Practice. High-alert medications in acute care settings [Internet]. US: Institute for Safe Medication Practices; 2018 [cited date 2021 December]. Available from: https://www.ismp.org/recommendations/high-alert-medications-acute-list.

2. da Silva JS, Seres DS, Sabino K, Adams SC, Berdahl GJ, Citty SW, et al. ASPEN consensus recommendations for refeeding syndrome. Nutr Clin Pract. 2020; 35: 178-195.

3. Erratum: Joshua S. V. da Silva. ASPEN Consensus Recommendations for Refeeding Syndrome. Nutr Clin Pract. 2020;35(2):178-195. Nutr Clin Pract. 2020; 35: 584-585.

4. Mirtallo JM, Ayers P, Boullata J, Gura KM, Plogsted S, Worthington P, et al. ASPEN lipid injectable emulsion safety recommendations, part1: Background and adult considerations. Nutr Clin Pract. 2020; 35: 769-782.

5. Worthington P, Gura KM, Kraft MD, Nishikawa R, Guenter P, Sacks GS, et al. Update on the use of filters for parenteral nutrition: An ASPEN position paper. Nutr Clin Pract. 2021; 36: 29-39.

6. Christensen ML, Ayers P, Boullata JI, Guenter P, Gura KM, Holcombe B, et al. Lipid injectable emulsion survey with gap analysis. Nutr Clin Pract. 2017; 32: 694-702.

7. Robinson DT, Ayers P, Fleming B, Gura KM, Gutsul L, Michalski A, et al. Recommendations for photoprotection of parenteral nutrition for premature infants: An ASPEN position paper. Nutr Clin Pract. 2021; 36: 927-941.

8. Cober MP, Gura KM, Mirtallo JM, Ayers P, Boullata J, Anderson CR, et al. ASPEN lipid injectable emulsion safety recommendations part 2: Neonate and pediatric considerations. Nutr Clin Pract. 2021; 36: 1106-1125.

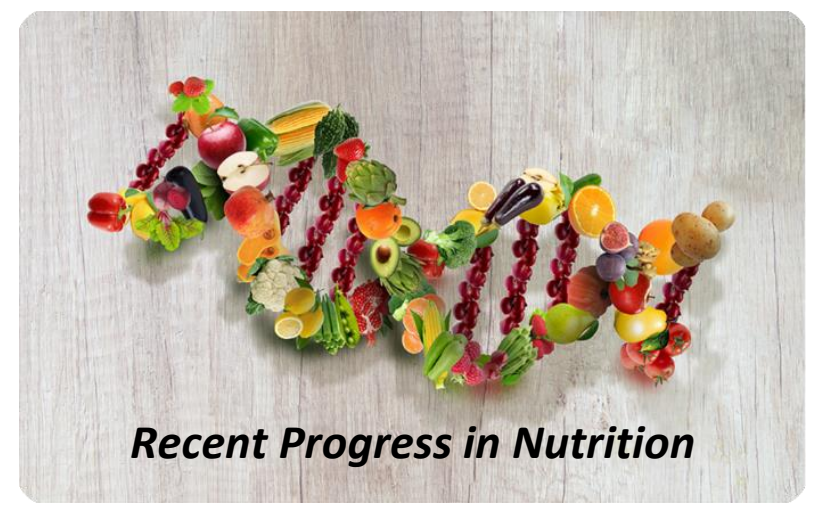

Enjoy Recent Progress in Nutrition by:

1. Submitting a manuscript

2. Joining in volunteer reviewer bank

3. Joining Editorial Board

4. Guest editing a special issue

For more details, please visit: http://www.lidsen.com/journals/rpn 